\title{
REMARKS ON SUPERSTRING AMPLITUDES IN HIGHER GENUS
}

\author{
RICCARDO SALVATI MANNI
}

\begin{abstract}
Very recently, Grushevsky continued D'Hoker and Phong's program of finding the chiral superstring measure from first principles by constructing modular forms satisfying certain factorization constraints. He has proposed an ansatz in genus 4 and conjectured a possible formula for the superstring measure in any genus, subject to the condition that certain modular forms admit holomorphic roots. In this note we want to give some evidence that Grushevsky 's approach seems to be very fruitful.
\end{abstract}

\section{INTRODUCTION}

D'Hoker and Phong managed, in a sequence of papers, to compute the genus 2 superstring measure from first principles in terms of modular forms cf. [4], [5], [6],[7] and verified some corresponding results such as the vanishing cosmological constant, 2-3- point scattering amplitudes et cetera. Since a derivation in higher genus from first principles appears prohibitively difficult at the present time, as an alternative approach, they suggested looking for ansatze satisfying factorization constraints consistent with the genus 2 formula, and in particular a certain set of conditions for an eventual genus 3 ansatz of .[8] and [9].

Cacciatori, Dalla Piazza, and van Geemen, cf. [1] proposed an ansatz for the chiral superstring measure in genus 3 in which they relaxed one of the conditions imposed by D'Hoker and Phong. This relaxing appears necessary otherwise the solution to the constraints of D'Hoker and Phong does not exist. Very recently Grushevsky rewrote the ansatz of D'Hoker and Phong in genus 2, and of Cacciatori, Dalla Piazza, and van Geemen in genus 3 in terms of modular forms associated to isotropic spaces of theta characteristics. This allows him to give a straightforward generalization of the chiral superstring measure to higher genera, which for genus 4 is an appropriate holomorphic modular form . For higher genera he conjectures a possible ansatz, satisfying the factorization constraints, but involving holomorphic roots. of suitable monomials in the theta constants. In this note we want to give

Date: June 16, 2021. 
some evidence that Grushevsky's approach seems to be the more fruitful. In fact we shall consider properties of products of theta constants with characteristics forming even cosets of isotropic subspaces. We prove that in genus 5, Grushevsky's ansatz, that involves square roots of theta constants is holomorphic on $\mathcal{M}_{5}(1,2)$. Another interesting results that we will get is to verify, without any tedious computation, that Grushevsky's proposed ansatz satisfies further physical constraints, for example that it is easy to prove the vanishing of cosmological constant when $g \leq 4$.

\section{Notations, Definitions And BAsic facts}

We denote by $\mathcal{H}_{g}$ the Siegel upper half-space of symmetric complex matrices with positive-definite imaginary part, called period matrices. The action of the symplectic group $\operatorname{Sp}(g, \mathbb{Z})$ on $\mathcal{H}_{g}$ is given by

$$
\left(\begin{array}{ll}
A & B \\
C & D
\end{array}\right) \circ \tau:=(C \tau+D)^{-1}(A \tau+B)
$$

where we think of elements of $\operatorname{Sp}(g, \mathbb{Z})$ as of consisting of four $g \times g$ blocks, and they preserve the symplectic form given in the block form as $\left(\begin{array}{cc}0 & 1 \\ -1 & 0\end{array}\right)$.

For a period matrix $\tau \in \mathcal{H}_{g}, z \in \mathbb{C}^{g}$ and $\varepsilon, \delta \in \mathbb{F}_{2}^{g}$ (where $\mathbb{F}_{2}$ denotes the abelian group $\mathbb{Z} / 2 \mathbb{Z}=\{0,1\}$ ) the associated theta function with characteristic $m=[\varepsilon, \delta]$ is

$\theta_{m}(\tau, z)=\theta\left[\begin{array}{l}\varepsilon \\ \delta\end{array}\right](\tau, z)=\sum_{n \in \mathbb{Z}^{g}} \exp \left(\pi i\left(\tau[(n+\varepsilon / 2)]+2 t(n+\varepsilon / 2)^{\prime}(z+\delta / 2)\right)\right.$.

Here we denote by $X^{\prime}$ taking the transpose of $X$ and $A[X]:=X^{\prime} A X$ As a function of $z, \theta_{m}(\tau, z)$ is odd or even depending on whether the scalar product $\varepsilon \cdot \delta \in \mathbb{F}_{2}$ is equal to 1 or 0 , respectively.

Theta constants are restrictions of theta functions to $z=0$. All odd theta constants vanish identically in $\tau$. We define the level subgroups of $\operatorname{Sp}(g, \mathbb{Z})$ as follows:

$$
\begin{gathered}
\Gamma_{g}(n):=\left\{\gamma=\left(\begin{array}{ll}
A & B \\
C & D
\end{array}\right) \in \Gamma_{g} \mid M \equiv\left(\begin{array}{ll}
1 & 0 \\
0 & 1
\end{array}\right) \bmod n\right\} \\
\Gamma_{g}(n, 2 n):=\left\{\gamma \in \Gamma_{g}(n) \mid \operatorname{diag}\left(A B^{\prime}\right) \equiv \operatorname{diag}\left(C D^{\prime}\right) \equiv 0 \bmod 2 n\right\} .
\end{gathered}
$$

Here $\operatorname{diag}(X)$ means the vector consisting of the diagonal entries of the square matrix $X$. These are normal subgroups of $\operatorname{Sp}(g, \mathbb{Z})$ for $n$ even.

We shall write $\Gamma_{g}$ for

$$
\Gamma_{g}(1)=\operatorname{Sp}(g, \mathbb{Z})
$$


Let $k$ be a positive integer and $\Gamma$ be a subgroup of finite index in $\Gamma_{g}$ A multiplier system of weight $k / 2$ for $\Gamma$ is a map $v: \Gamma \rightarrow \mathbb{C}^{*}$, such that the map

$$
\gamma \mapsto v(\gamma) \operatorname{det}(C \tau+D)^{k / 2}
$$

satisfies the cocycle condition for every $\gamma \in \Gamma$ and $\tau \in \mathcal{H}_{g}$.

With these notations, we say that a holomorphic function $f$ defined on $\mathcal{H}_{g}$ is a modular form of weight $k / 2$ with respect to $\Gamma$ and $v$ if

$$
f(\gamma \circ \tau)=v(\gamma) \operatorname{det}(C \tau+D)^{k / 2} f(\tau) \quad \forall \gamma \in \Gamma, \forall \tau \in \mathcal{H}_{g}
$$

and if additionally $f$ is holomorphic at all cusps when $g=1$ We denote by $[\Gamma, k / 2, v]$ the vector space of such functions. We omit the multiplier if it is trivial.

The full symplectic group acts on theta constants with characteristics as follows:

$$
\begin{gathered}
\theta_{\gamma \cdot m}(\gamma \cdot \tau)= \\
\kappa(\gamma) \exp \left((1 / 4) \pi i\left(B^{\prime} D[\varepsilon]-2 \varepsilon^{\prime} B^{\prime} C \delta+A^{\prime} C[\delta]-2 \operatorname{diag}\left(A B^{\prime}\right)(D \varepsilon-C \delta)\right)\right) \\
\operatorname{det}(C \tau+D)^{\frac{1}{2}} \theta_{m}(\tau),
\end{gathered}
$$

where $\kappa(\gamma)$ is an eighth root of unity, and the action on the characteristic is

$$
\gamma \cdot m=\gamma\left(\begin{array}{l}
\varepsilon \\
\delta
\end{array}\right):=\left(\begin{array}{cc}
D & -C \\
-B & A
\end{array}\right)\left(\begin{array}{l}
\varepsilon \\
\delta
\end{array}\right)+\left(\begin{array}{l}
\operatorname{diag}\left(C D^{\prime}\right) \\
\operatorname{diag}\left(A B^{\prime}\right)
\end{array}\right)
$$

where the addition in the right-hand-side is taken in $\mathbb{F}_{2}^{2 g}$.

We observe at a certain point we shall consider the addition in the right-hand-side is taken in $\mathbb{Z}^{2 g}$

In [13] it is proved that theta constants are modular forms of weight $1 / 2$ with respect to $\Gamma_{g}(4,8)$.

The action of $\Gamma_{g}(2)$ on the set of theta constants produces a multiplier. Let $M=\left(m_{1}, m_{2}, \ldots, m_{k}\right)$ be a sequence of characteristics, we set

$$
P(M)=\prod_{i=1}^{k} \theta_{m_{i}}
$$

For $k=2 r$ even, in the previous discussion we get that that $P(M)$ is a modular $m$ with respect to $\Gamma_{g}(2)$ and a suitable character. From [12], we know that the character is trivial if the matrix $M$ satisfies the congruences

$$
\begin{gathered}
M^{\prime} M \equiv r\left(\begin{array}{cc}
0 & 1_{g} \\
1_{g} & 0
\end{array}\right) \quad \bmod 2 \\
\operatorname{diag}\left(M^{\prime} M\right) \equiv 0 \quad \bmod 4
\end{gathered}
$$


For all characteristic $m=[\varepsilon, \delta]$ and $n=\left(\varepsilon_{1}, \delta_{1}\right)$, we set

$$
e(m, n)=(-1)^{\varepsilon \cdot \delta_{1}+\varepsilon_{1} \cdot \delta} \text {. }
$$

We say that a subspace $V \subset \mathbb{F}_{2}^{2 g}$ is isotropic if $e(m, n)=1$ for all $m, n$ in $V$. A maximal isotropic space has dimension $g$. Each isotropic subspace has even cosets, i.e cosets all of which entries are even characteristics. For details we refer to [16]. Let $V+m$ be an even coset of an isotropic $j$ dimensional space. Let us assume that $2^{j} s=2^{k} \geq 8$, thus the form $P(V+m)^{s}$ belongs to $\left[\Gamma_{g}(2), 2^{k-1}\right]$. for details we refer to[14] and [16].

One can thus study the orbits of characteristics or sets of characteristics under the symplectic group action. This was done in [15] [17], where, in particular, it can be found that $\operatorname{Sp}\left(g, \mathbb{F}_{2}\right)$ acts transitively on the even cosets of isotropic $j$ dimensional spaces.

Moreover $\Gamma_{g}(1,2) / \Gamma_{g}(2)$ acts transitively on even isotropic $j$ dimensional spaces. As consequence of these facts we get the following

Lemma 1. Let us assume that 8 divides $2^{i} s$ and $g \geq i$, thus the form

$$
S_{i, s}^{g}=\sum_{V+m} \varepsilon_{V+m} P(V+m)^{s}(\tau)
$$

belongs to $\left[\Gamma_{g}, 2^{k-1}\right]$ providing that we are summing over all even cosets of all $i$ dimensional even isotropic spaces and $\varepsilon_{V+m}$ are suitable signs .

Proof Since $\operatorname{Sp}\left(g, \mathbb{F}_{2}\right)$ acts transitively on the set of even cosets of isotropic spaces of the same dimension, we will sum over the associated monomials in the theta constants using the transformation formula. Thus we can start from a fixed sequence $M$, corresponding to the vectors of a even totally isotropic space $V$. let $G_{V}$ be the stabilizer of $V$ in $\operatorname{Sp}\left(g, \mathbb{F}_{2}\right)$, thus from the transformation formula, [17] we get that

$$
\frac{1}{\left|G_{V}\right|} \sum_{\gamma \in G} \operatorname{det}(C \tau+D)^{-2^{k-1}} P(M)^{s}(\gamma \cdot \tau)=\sum_{V+m} \varepsilon_{W+m} P(W+m)^{s}(\tau)
$$

with

$$
\begin{gathered}
\varepsilon_{W+m}=\exp \left(( 1 / 4 ) \pi i \left(\operatorname{tr}\left(A B^{\prime}\left[M_{1}\right]+C D^{\prime}\left[M_{2}\right]\right)\right.\right. \\
\exp \left(( 1 / 2 ) \pi i \left(\operatorname{tr}\left(C B^{\prime} M_{1} M_{2}^{\prime}+\left(\gamma^{-1} M\right)_{1}^{\prime}\left(N-\gamma^{-1} M\right)_{2}\right)\right.\right.
\end{gathered}
$$

Here we denoted with $W+m$ the even coset obtained by $V$ acting with $\gamma^{-1}$. This means that if $M=\left(\begin{array}{l}M_{1} \\ M_{2}\end{array}\right)$ is the sequence of vectors in $V, \gamma^{-1} M$ is the sequence of vectors in $W+m$ with coefficients in $\mathbb{F}_{2}$. Here $N$ is a sequence of vectors in $\mathbb{Z}^{2 g}$ induced by the action of $\Gamma_{g}$ on $M$ with the addition in $\mathbb{Z}$. Obviously we have $N \equiv \gamma^{-1} M \bmod 2$. 
By construction this form belongs necessarily to $\left[\Gamma_{g}, 2^{i-1} s\right]$. A priori it could be identically zero. The congruences satisfied by the matrix $s M$, imply that $\varepsilon_{V}= \pm 1$.

We can refine the previous result proving an interesting result and a consequent corollary that result to be fundamental in [11]

Proposition 2. Let us assume that 16 divides $2^{i} s$ and $g \geq i$,

$$
S_{i, s}^{g}=\sum_{V+m} P(V+m)^{s}(\tau)
$$

belongs to $\left[\Gamma_{g}, s 2^{j-1}\right]$ provided that we are summing over all even cosets of $i$ dimensional isotropic spaces

Proof We have to prove that in these cases $\varepsilon_{V}=1$. In fact the matrix $s M$ satisfies the congruences

$$
\begin{aligned}
& { }^{t} M M \equiv\left(\begin{array}{ll}
0 & 0 \\
0 & 0
\end{array}\right) \quad \bmod 4 \\
& \operatorname{diag}\left({ }^{t} M M\right) \equiv 0 \quad \bmod 8 .
\end{aligned}
$$

An immediate computation shows that from the previous formula we have possibly non trivial term only when $s=1$ ( hence $\operatorname{dim} V \geq 4$ ). This is

$$
\exp \left(( 1 / 2 ) \pi i \left(\operatorname{tr}\left(\left(\gamma^{-1} M\right)_{1}^{\prime}\left(N-\gamma^{-1} M\right)_{2}\right)\right.\right.
$$

Now to compute this term we can use a set of generators of the group $\operatorname{Sp}\left(g, \mathbb{F}_{2}\right)$ or just observe that in [14] the case of $\mathrm{i}=4$ for the action of $\Gamma_{g}$ was considered. In this case appear all even coset of four dimensional totally isotropic spaces and the coefficients are all 1. Since an even coset of a isotropic subspace $V$ of dimension $j+1$ is union of two even cosets of a suitable isotropic subspace $W$ of dimension $j$, by induction we see that the above term is always 1 . In fact if

$$
V+m=(W+m) \cup(W+n)
$$

we have that

$$
\varepsilon_{V+m}=\varepsilon_{W+m} \varepsilon_{W+n}=1 .
$$

As consequence of the above proposition we get the following corollary stated also in[11]

Corollary 3. Let us assume that 16 divides $2^{i} s$,

$$
P_{i, s}^{g}(\tau)=\sum_{V} P(V)^{s}(\tau)
$$

belongs to $\left[\Gamma_{g}(1,2), 2^{i-1} s\right]$ provided that we are summing over $j$ dimensional even isotropic spaces 
Remark 4. A similar statement holds even if 8 divides $2^{i} s$, but we will get some signs. To avoid confusion, from now on we assume that 16 divides $2^{i} s$.

We observe that $P_{i, s}^{g}(\tau)$ can be considered also as the partial sum of $S_{i, s}^{g}(\tau)$ where we restrict our computation to all even cosets of $j$ dimensional even isotropic spaces containing the 0 characteristic. Thus similarly to $P_{i, s}^{g}(\tau)$, for every even characteristic $m$, we can define $P_{i, s}^{g}[m](\tau)$ where we take the sum over even cosets cosets containing the characteristic $m$. Obviously $P_{i, s}^{g}[0](\tau)=P_{i, s}^{g}(\tau)$ and

$$
2^{i} S_{i, s}^{g}(\tau)=\sum_{m e v e n} P_{i, s}^{g}[m](\tau)
$$

We observe that the coefficient $2^{i}$ of $S_{i, s}^{g}$ is due to the fact that in the summation in the right hand side an even coset of a $i$ dimensional subspace appear with multeplicity $2^{i}$, i.e. the number of vectors in the coset.

Obviously $P_{i, s}^{g}[m](\tau)$ are modular form with respect to a subgroup of $\Gamma_{g}$ conjugated to $\Gamma_{g}(1,2)$. We recall that $\Gamma_{g}(1,2)$ is not normal in $\Gamma$.

\section{Grushevsky's Results}

In this short section we recall the main results obtained in [11]. Obviously we can assume that 16 divides $2^{i} s$. All details can be found in the above cited paper.

The modular forms $P_{i, s}^{g}(\tau)$ has the following remarkable property

Proposition 5. The modular forms $P_{i, s}^{g}$ restrict to the locus of block diagonal period matrices $\mathcal{H}_{k} \times \mathcal{H}_{g-k}$ as follows

$$
P_{i, s}^{g}=\sum_{0 \leq n, m \leq i \leq n+m} N_{n, m ; i} P_{n, 2^{i-n} s}^{k} P_{m, 2^{i-m} s}^{g-k}
$$

with

$$
N_{n, m ; i}=\prod_{j=0}^{n+m-i-1} \frac{\left(2^{n}-2^{j}\right)\left(2^{m}-2^{j}\right)}{2^{n+m-i}-2^{j}}
$$

These forms are the basic bricks for an easy writing of the low genus superstring scattering amplitudes proposed by D'Hoker and Phong [4] for genus 2 and by Cacciatori, Dalla Piazza, and van Geemen [1] for genus 3 . In fact in [11] has been proved 
Theorem 6. For $g \leq 4$ the function

$$
\Xi^{(g)}[0]:=\frac{1}{2^{g}} \sum_{i=0}^{g}(-1)^{i} 2^{\frac{i(i-1)}{2}} P_{i, 2^{4-i}}^{g}
$$

is a modular form of weight 8 with respect to $\Gamma_{g}(1,2)$, such that its restriction to $\mathcal{H}_{k} \times \mathcal{H}_{g-k}$ is equal to $\Xi^{(k)}[0] \cdot \Xi^{(g-k)}[0]$.

Thus these are natural candidates for the superstring measures.

Moreover in [11], it is shown that the above statement holds for gi4 as well, up to a possible inconsistency in the choice of the roots.

\section{EvidenCES FOR THE ANSATZ}

In this section we shall give evidence for these natural candidates verifying that the proposed ansatz satisfies further physical constraints.

Similarly to the $P^{\prime} s$ case we define

$$
\Xi^{(g)}[m]=\frac{1}{2^{g}} \sum_{i=0}^{g}(-1)^{i} 2^{\frac{i(i-1)}{2}} P_{i, 2^{4-i}}^{g}[m]
$$

Summing up we get

\section{Lemma 7.}

$$
\Xi^{(g)}=\sum_{m} \Xi^{(g)}[m]=\frac{1}{2^{g}} \sum_{i=0}^{g}(-1)^{i} 2^{\frac{i(i+1)}{2}} S_{i, 2^{4-i}}^{g}
$$

Showing that the cosmological constant vanishes is equivalent to show that $\Xi^{(g)}$ is identically zero on the moduli space of curves $\mathcal{M}_{g}$ This has been verified for genus 2 in [4] and for genus 3 in [1]. We shall prove it in genus 4 .

This is a consequence of remarkable formulas proved around thirty years ago [14]. In fact in the above cited paper Igusa introduced modular forms of weight 8 related to $\Gamma_{g}$ and relations among them.

Lemma 8. We have

$$
\begin{gathered}
\left(2^{2 g}-1\right) S_{0,16}=6 S_{1,8}+24 S_{2,4} \\
\left(2^{2 g-2}-1\right) S_{1,8}=18 S_{2,4}+168 S_{3,2} \\
\left(2^{2 g-4}-1\right) S_{2,4}=42 S_{3,2}+840 S_{4,1}
\end{gathered}
$$

for $g \geq 2,3,4$ respectively

As immediate consequence of the lemma we get the following 
Theorem 9. The modular form $\Xi^{(g)}$ is identically zero on $\mathcal{M}_{g}$ for $g \leq 4$

Proof We shall prove only the case $g=4$. The other are similar and easier According to our previous discussions we have

$$
2^{4} \Xi^{(4)}=S_{0,16}-2 S_{1,8}+8 S_{2,4}-64 S_{3,2}+1024 S_{4,1}
$$

From previous lemma we get

$$
\Xi^{(4)}=2^{2} \cdot 3 \cdot 7^{-1}\left(15 S_{0,16}-2 S_{1,8}\right)
$$

According to theorem 1 in [14], we get that

$$
\Xi^{(4)}=-2^{8} \cdot 3^{3} \cdot 5 J(\tau)
$$

Here $J(\tau)$ is Schottky's polynomial that vanishes along $\mathcal{M}_{4}$.

We end our discussion, considering the proposed ansatz for the superstring measure in any genus, in the genus 5 case. In this case the theorem involves square root of modular forms.

$$
\Xi^{(5)}[0]:=\frac{1}{2^{5}} \sum_{i=0}^{5}(-1)^{i} 2^{\frac{i(i-1)}{2}} P_{i .2^{4-i}}^{5}
$$

involves the term $P_{5,1 / 2}^{5}$. Now the problem of the definition of square roots of theta constants on suitable covering of $\mathcal{M}_{g}$ has been settled in [19]. First of all let us define $\mathcal{M}_{g}(n, 2 n)$ as the inverse image of $\mathcal{M}_{g}$ in $\mathcal{H}_{g} / \Gamma(n, 2 n)$ under the standard projection map. $\sqrt{\theta_{m}}$ can be considered as a modular form on $\mathcal{M}_{g}(4,8)$, in fact the divisor of theta constants are tangent to $\mathcal{M}_{g}(4,8)$ and this allows to have square roots. The main problem involves the factor of automorphy of the transformation formula, in fact for any subgroup of finite index in $\Gamma_{g}$ the $\sqrt{\theta_{m}}$ have diffent multipliers. These obstruction can be eliminated in some special case, in fact as consequence of relation of Schottky type in [19] it has been proved

Lemma 10. Let $M$ be a isotropic 3 dimensional space, then for all ( even) cosets $M+m$

$$
\sqrt{P(M+m)}
$$

belong to the same automorphy factor in $\mathcal{M}_{g}(4,8)$

Now let us assume $g=5$. Even cosets of maximal isotropic spaces are only isotropic spaces. Let $V$ be a maximal 5 dimensional even isotropic space, Since $V=M \cup M+n_{1} \cup M+n_{2} \cup M+n_{3}$ for a suitable 3 dimensional, isotropic space $M$. Hence the factor of automorphy of 
the term $\sqrt{P(V)}$ appearing in $P_{5,1 / 2}^{5}$ is the fourth power of $\sqrt{P(W)}$ where $W \subset V$ is a three dimensional isotropic space. First of all we want to prove that all $\sqrt{P(V)}, V$ even maximal isotropic space have the same factor of automorphy. This can be reduced to a combinatorial

Lemma 11. Let $V, Z$ be even maximal isotropic space in genus 5 then there exist a sequence of even maximal isotropic space $V_{0}=V, V_{1}, \ldots, V_{n}=$ $Z$ and a sequence of even 3 dimensional isotropic space $M_{1}, \ldots, M_{n}$ such that

$$
M_{i} \subset V_{i-1} \cap V_{i}
$$

Proof Let $x_{1}, \ldots x_{10}$ be the coordinates of $\mathbb{F}_{2}^{10}$, without any loss of generality ( all the other cases are easier) we can assume that $V=V_{0}$ is defined by $\left\{x_{1}=x_{2}=x_{3}=x_{4}=x_{5}=0\right\}$ and $Z=V_{n}$ is defined by $\left\{x_{6}=x_{7}=x_{8}=x_{9}=x_{10}=0\right\}$. Let $M_{1}$ be the subspace of $V_{0}$ defined by $\left\{x_{6}=x_{7}=0\right\}$.

$M_{1}$ is obviously contained in the subspace $V_{1}$ defined by $\left\{x_{3}=x_{4}=\right.$ $\left.x_{5}=x_{6}=x_{7}=0\right\}$. Let $M_{2}$ be the subspace of $V_{1}$ defined by $\left\{x_{8}=\right.$ $\left.x_{9}=0\right\} . M_{2} \subset V_{2} V_{2}$ is defined by $\left\{x_{5}=x_{6}=x_{7}=x_{8}=x_{9}=0\right\}$ Now $M_{3} \subset V_{2}\left\{x_{10}=x_{4}=0\right\}$ Hence $M_{3} \subset V_{3}=Z$ As consequence of the above lemma we have

Theorem 12. $P_{5,1 / 2}^{5}$ is a modular form relative to $\Gamma_{5}(1,2)$, once we restrict to the $\mathcal{M}_{5}(1,2)$, hence $\Xi^{(5)}[0]$ is well defined on $\mathcal{M}_{5}(1,2)$.

Proof We restrict to the inverse image of $\mathcal{M}_{g}$ in $\mathcal{H}_{g}$. From the previous lemma, it follows that all terms appearing in $P_{5,1 / 2}^{5}$ have the same factor of automorphy w.r.t $\Gamma_{5}(4,8)$. In fact the factor of automorphy of $P(V)$ is the fourth power of the factor of automorphy of $P\left(M_{1}\right)$. But this is the same factor of automorphy of $P\left(V_{1}\right)$. Now we can iterate the process . The factor of automorphy of $P\left(V_{1}\right)$ is the forth power of the factor of automorphy of $P\left(M_{2}\right)$. But this is the same factor of automorphy of $P\left(V_{2}\right)$ an so on.

By transformation formula this implies that the factor of automorphy is always the same also at the $\Gamma_{5}(2)$ level. The action of the group $\Gamma_{5}(1,2) / \Gamma_{5}(2)$ produces the modular form $P_{5,1 / 2}^{5}$. Hence $P_{5,1 / 2}^{5}$ is a modular form wrt $\Gamma_{5}(1,2)$ with possibly a character induced by the square root .

We observe that the previous lemma holds even if $V$ and $Z$ are even 4 dimensional maximal isotropic space in genus 5 . Now we can repeat the same argument with terms $\sqrt{P(W)}$ where $W$ is even 4 dimensional isotropic space in genus 5 . Hence $\sqrt{P(W)}$ and $\sqrt{P(W+m)}$ have the same factor of automorphy, here $W+m$ is an even coset. Since we 
have always a decomposition $V=W \cup W+m$, we have the character appearing in the transformation formula for $\sqrt{P(V)}$ is the square of the character appearing in the transformation formula for $\sqrt{P(W)}$. From Lemma 1 , we know that $P(W)$ has trivial character, hence $\sqrt{P(V)}$ has trivial character.

Since similar results hold for $\Xi^{(5)}[\mathrm{m}]$, we get

Corollary 13. $\Xi^{(5)}$ is well defined on $\mathcal{M}_{5}$

\section{FURTHER REMARKS}

Because of Grushevsky's result, it seems reasonable to consider also the modular forms $S_{i, s}^{g}(\tau)$ without any restriction on the weight. In this section we shall show that they have some nice properties as modular forms. For doing this we need to introduce the Siegel $\Phi$-operator. For $f: H_{g} \rightarrow \mathbb{C}$ holomorphic we set formula

$$
\Phi(f)\left(\tau_{1}\right)=\lim _{\lambda \longrightarrow+\infty} f\left(\begin{array}{cc}
\tau_{1} & 0 \\
0 & i \lambda
\end{array}\right)
$$

for all $\tau_{1} \in \mathcal{H}_{g-1}$. In particular this operator maps $\left[\Gamma_{g}, k,\right]$ to $\left[\Gamma_{g-1}, k\right]$ and $\left[\Gamma_{g}(n, 2 n), k\right]$ to $\left[\Gamma_{g-1}(n, 2 n), k\right]$. This operator has a relevant importance in the theory of modular forms, we refer to [13] or [10] for details. In the case of the full modular group, a cusp form is a modular form that is in the kernel of the $\Phi$ operator. In the case of subgroup of the modular group, a modular form is a cusp form if all its conjugate with respect to the $\Gamma_{g}$ action are in the kernel of the $\Phi$ operator. For any of such groups, we shall denote by $[\Gamma, k]_{0}$ the subspace of cusp forms.

The image $\Phi\left(\theta_{m}\right)$ can be easily computed. I Indeed if $m=[\varepsilon, \delta]$ be an even characteristic with $\varepsilon=\left(\varepsilon^{\prime}, \varepsilon_{g}\right)=\left(\varepsilon_{1}, \varepsilon_{2}, \ldots \varepsilon_{g}\right)$ and similarly $\delta=\left(\delta^{\prime}, \delta_{g}\right)=\left(\delta_{1}, \delta_{2}, \ldots \delta_{g}\right)$, then we have

$$
\Phi\left(\theta_{m}\right)\left(\tau_{1}\right)=\left\{\begin{array}{l}
\theta_{m^{\prime}}\left(\tau_{1}\right) \text { if } \varepsilon_{g}=0, \\
0 \text { if } \varepsilon_{g}=1
\end{array}\right.
$$

Here $m^{\prime} \in \mathbb{F}_{2}^{2 g-2}$ is the characteristic obtained by $m$ deleting the entries $\varepsilon_{g}$ and $\delta_{g}$.

We want to apply the $\Phi$ operator to the modular forms $S_{i, s}^{g}(\tau)$. As consequence of simple computations or even as consequence of Grushevsky's result, we get

Lemma 14. Let us assume that $0 \leq i \leq g$ and 16 divides $2^{i} s$, then 


$$
\Phi\left(S_{i, s}^{g}(\tau)\right)=2^{i+1} S_{i, s}^{g-1}+S_{i-1,2 s}^{g-1}
$$

when $0<i<g$.

For $i=g$ we have

$$
\Phi\left(S_{g, s}^{g}(\tau)\right)=S_{g-1,2 s}^{g-1}
$$

and for $i=0$ we have

$$
\Phi\left(S_{0, s}^{g}(\tau)\right)=2 S_{0, s}^{g-1}
$$

For $2^{k} s$ divisible by $\max \left(16,2^{g}\right)$ we define

$$
\Xi_{2^{k} s}^{(g)}=\sum_{m} \Xi_{2^{k} s}^{(g)}[m]=\frac{1}{2^{g}} \sum_{i=0}^{g}(-1)^{i} 2^{\frac{i(i+1)}{2}} S_{i, s 2^{k-i}}^{g}
$$

As immediate consequence we get

Theorem 15. For any $g$, let us assume that $\max \left(16,2^{g}\right)$ divides $2^{k} s$, then $\Xi_{2^{k} s}^{(g)}$ is a cusp form.

Proof This is also an immediate consequence of the previous results, in fact we have

$$
\begin{gathered}
\Phi\left(\Xi_{2^{k} s}^{(g)}\right)=\frac{1}{2^{g}} \sum_{i=0}^{g}(-1)^{i} 2^{\frac{i(i+1)}{2}} \Phi\left(S_{i, 2^{k-i} s}^{g}\right)= \\
\frac{1}{2^{g}} \sum_{i=1}^{g-1}(-1)^{i} 2^{\frac{i(i+1)}{2}}\left(2^{i+1} S_{i, 2^{k-i} s}^{g-1}+S_{i-1,2^{k-i+1} s}^{g-1}\right)+ \\
\frac{(-1)^{g}}{2^{g}} 2^{\frac{g(g+1)}{2}} S_{g-1,2^{k-g+1} s}^{g-1}= \\
\left.\frac{1}{2^{g}}\left(\sum_{i=2}^{g}(-1)^{i+1} 2^{\frac{i(i+1)}{2}} S_{i-1,2^{k-i+1} s}^{g-1}+\sum_{i=1}^{g-1}(-1)^{i} 2^{\frac{i(i+1)}{2}} S_{i-1,2^{k-i+1} s}^{g-1}\right)\right)+ \\
\frac{1}{2^{g}}\left(2 S_{0,2^{k} s}^{g-1}+(-1)^{g} 2^{\frac{g(g+1)}{2}} S_{g-1,2^{k-g+1} s}^{g-1}\right)=0
\end{gathered}
$$

Since we get different signs for the same modular form.

Remark 16. From a discussion with Sam Grushevsky, we realized that an alternative proof of this result, more in the spirit of this paper, is possible. According to Grushevsky's result we have that the modular forms $\Xi_{2^{k} s}^{(g)}$ restricts on $\mathcal{H}_{g-1} \times \mathcal{H}_{1}$ as $\Xi_{2^{k} s}^{(g-1)} \cdot \Xi_{2^{k} s}^{(1)}$.

So, applying the $\Phi$ operator is equivalent to compute

$$
\lim _{\lambda \rightarrow+\infty} \Xi_{2^{k} s}^{(g-1)}\left(\tau_{1}\right) \cdot \Xi_{2^{k} s}^{(1)}(i \lambda)=
$$




$$
\lim _{\lambda \rightarrow+\infty}\left(S_{0,2^{k} s}^{1}-2 S_{1,2^{k-1} s}^{1}\right) \Xi_{2^{k} s}^{(g-1)}\left(\tau_{1}\right)
$$

Now a simple computation tells us that

$$
\lim _{\lambda \rightarrow+\infty}\left(S_{0,2^{k} s}^{1}-2 S_{1,2^{k-1} s}^{1}\right)=0
$$

As an immediate consequence of the previous theorem, we can reformulate the result of Theorem 10 , in fact we have following

Corollary 17. For $g \leq 4$, the cosmological constant $\Xi^{(g)}=\Xi_{16}^{(g)}$ vanishes along $\mathcal{M}_{g}$

Proof For $g \leq 4, \Xi^{(g)}$ is a cusp form of weight 8. It is a well known fact that $\left[\Gamma_{g}, 8\right]_{0}=0$ for $g \leq 3$. Moreover the space $\left[\Gamma_{4}, 8\right]_{0}$ is one dimensional and it is generated by the Schottky polynomial $J(\tau)$, cf.[18].

\section{Non EXIstence of D'Hoker Phong FORM IN Genus 3}

As we state in the introduction, Cacciatori, Dalla Piazza, and van Geemen, cf. [1] proposed an ansatz for the chiral superstring measure in genus 3 relaxing a condition posed by D' Hoker and Phong. We shortly explain why this relaxing is necessary. This is not a proof. A proof has been announced in [1] and it will appear in [2]. The solution proposed by $\mathrm{D}^{\prime}$ Hoker and Phong should include a factor $\theta_{0}^{4}$. This would imply that the proposed ansatz for the superstring measure should be $\theta_{0}^{4} \Xi_{6}[0]$ with $\Xi_{6}[0]$ a modular form of weight 6 w.r.t $\Gamma_{g}(1,2)$ and a suitable character. This will be the same character $\chi_{g}$ appearing in the transformation formula of $\theta_{0}^{4}$, hence it is trivial when we restrict to $\Gamma_{g}(2)$ Now when $g \leq 3$ the space of modular forms $\left[\Gamma_{3}(2), 6\right]$ is generated by monomials in the theta constant, that are particular theta series with characteristics. The same is true for their subspaces. In particular we have that theta series related to unimodular lattices of degree 12 are modular forms in $\left[\Gamma_{g}(1,2), 6, \chi_{g}\right]$. Now according to the table in [3] we have three integral classes of such lattices, namely $I_{12}$, $E_{8}+I_{4}$ and $D_{12}^{+}$, to these lattices ,up to multiplicative constants, are associated the forms $F_{1}, F_{3}$ and $F_{2}$ in the notation of [1]. These forms are linearly independent already in genus 2 , thus we have

$$
\operatorname{dim}\left[\Gamma_{2}(1,2), 6, \chi_{2}\right]=\operatorname{dim}\left[\Gamma_{3}(1,2), 6, \chi_{3}\right]=3
$$

Thus simple computations will produce that in genus 3 we cannot have the desidered restriction properties. 


\section{ACKnowledgements}

I am very thankful to Duong Phong, Sam Grushevsky and Bert van Geemen for several helpful comments to a preliminary version of this manuscript.

\section{REFERENCES}

[1] Cacciatori, S.L., Dalla Piazza, F., van Geemen, B.: Modular Forms and Three Loop Superstring Amplitudes. arXiv:0801.2543.

[2] Dalla Piazza, F., van Geemen, B.: to appear

[3] Conway, H., Sloane, N.J.A.:Sphere packings, lattices and groups Die Grundlehren der mathematischen Wissenschaften, Band 290, Springer: New York Berlin Heidelberg, 1988

[4] D'Hoker, E., Phong, D.H.: Two-Loop Superstrings I, Main Formulas. Phys. Lett. B 529 (2002) 241-255; hep-th/0110247.

[5] D'Hoker, E., Phong, D.H.: Two-Loop Superstrings II, The chiral Measure on Moduli Space. Nucl. Phys. B 636 (2002) 3-60; hep-th/0110283.

[6] D'Hoker, E., Phong, D.H.: Two-Loop Superstrings III, Slice Independence and Absence of Ambiguities. Nucl. Phys. B 636 (2002) 61-79; hep-th/0111016.

[7] D'Hoker, E., Phong, D.H.: Two-Loop Superstrings IV, The Cosmological Constant and Modular Forms. Nucl. Phys. B 639 (2002) 129-181; hep-th/0111040.

[8] D'Hoker, E., Phong, D.H.: Asyzygies, modular forms and the superstring measure I . Nucl. Phys. B $\mathbf{7 1 0 ~ ( 2 0 0 5 ) ; ~ h e p - t h / 0 4 1 1 1 5 9 ~}$

[9] D'Hoker, E., Phong, D.H.: Asyzygies, modular forms and the superstring measure II. Nucl. Phys. B 710 (2005); hep-th/0411182 .

[10] Freitag, E.: Siegelsche Modulfunktionen. Grundlehren der mathematischen Wissenschaften, Band 254 Berlin-Heidelberg-New York: Springer-Verlag. (1983)

[11] Grushevsky, S.:Superstring scattering amplitudes in higher genus arXiv: hepth/0803.3469

[12] Igusa, J.-I.: On the graded ring of theta-constants. Am. J. Math. 86 (1964), 219-246 .

[13] Igusa, J.-I.: Theta functions. Die Grundlehren der mathematischen Wissenschaften, Band 194. Springer-Verlag, New York-Heidelberg, 1972.

[14] Igusa, J.-I.: Schottky's invariant and quadratic forms E. B. Christoffel Int. Symp., Aachen (1981), 352-362 .

[15] Igusa, J.-I.: On Jacobi's derivative formula and its generalizations,Am. J. Math. 102 (1980), 409-446 .

[16] Salvati Manni, R.: On the dimension of the vector space $\mathbb{C}\left[\theta_{m}\right]_{4}$, Nagoya Math. J.

[17] Salvati Manni, R.: Thetanullwerte and stable modular forms, Amer. J. Math. 111 (1989), 435-455

[18] Salvati Manni, R : Modular forms of the fourth degree. (Remark on a paper of Harris and Morrison) Proc. Conf., Trento/Italy 1990, Lect. Notes Math. 1515 (1992), 106-111.

[19] Tsuyumine, S.: Thetanullwerte on a moduli space of curves and hyperelliptic loci Math. Z. 207 (1991), 539-568. 
Dipartimento di Matematica, Università "SapienzA", Piazzale A. Moro 2, Roma, I 00185, Italy

E-mail address: salvati@mat.uniroma1.it 